\title{
Development of a portable assembly-type cosmic-ray muon module for measuring the density structure of a column of magma
}

\author{
Hiroyuki K. M. Tanaka ${ }^{1}$, Tomohisa Uchida ${ }^{2}$, Manobu Tanaka ${ }^{3}$, Hiroshi Shinohara ${ }^{4}$, and Hideaki Taira ${ }^{1}$ \\ ${ }^{1}$ Earthquake Research Institute, the University of Tokyo, 1-1-1 Yayoi, Bunkyo, Tokyo 113-0032, Japan \\ ${ }^{2}$ Department of Physics, the University of Tokyo, 7-3-1 Hongo, Bunkyo, Tokyo 113-0033, Japan \\ ${ }^{3}$ Institute of Particle and Nuclear Studies, High Energy Accelerator Research Organization (KEK), \\ 1-1 Oho, Tsukuba, Ibaraki 305-0801, Japan \\ ${ }^{4}$ National Institute of Advanced industrial Science and Technology (AIST), \\ 1-1-1 Umezono, Tsukuba, Ibaraki 305-8568, Japan
}

(Received November 28, 2008; Revised May 15, 2009; Accepted June 5, 2009; Online published February 22, 2010)

\begin{abstract}
We have developed a portable assembly type cosmic-ray muon telescope system with power-effective real-time readings to monitor the internal structure of a volcano. Using this system, we have performed measurements at the Satsuma-Iojima volcano and studied the feasibility of using a continuous ux of cosmic-ray muons over the observation period. The system is based on the measurement of time-dependent muon absorption along different, nearly horizontal paths through a solid body. The rationale is that one can deduce the time-dependent changes in the density distribution of muon absorption in the interior of the object where an absorption variation, i.e., a density path variation, becomes an intensity variation since the muon energy spectrum is exponential or, expressed otherwise, it drops rapidly when the energy threshold increases. The muon telescope, which has a surface area of $1 \mathrm{~m}^{2}$, was installed at the observation point located $1.2 \mathrm{~km}$ from the summit crater of Satsuma-Iojima. Muon tracks within scintillator layers in the telescope were analyzed continuously by real-time three-dimensional image processing to measure the level of absorption along different ray paths through the summit crater region. A typical angular resolution of the muon detector of $\pm 16 \mathrm{mrad}$ corresponds to a spatial resolution of $\pm 20 \mathrm{~m}$ at a distance of $1.2 \mathrm{~km}$. Our results show the density structure determined in Satsuma-Iojima volcano, Japan, which is located above sea level. A density structure situated above sea level can be analyzed at a resolution that is signi cantly higher than is possible with conventional geophysical measurements.
\end{abstract}

Key words: Cosmic ray muon module, density path variation, time-dependent muon absorption, solid body, density structure.

\section{Introduction}

The muon is the most numerous of the charged elementary particles at sea level and is capable of traveling large distances. However, they lose energy in proportion to the amount of matter they pass through. Muons are highly penetrating, and a typical horizontally arriving cosmic ray muon with an energy of $1 \mathrm{TeV}$ penetrates $2.4 \mathrm{~km}$ of water (Table 1). When primary protons come into contact with the atmosphere, a proton-nucleus interaction takes place, which mainly produces pions, but also kaons. Charged pions and kaons decay into muons within a short time. The amount of energy lost by muons in the atmosphere is considerably small and, consequently, all muons in the vertical direction with energy above a few $\mathrm{GeV}$ will reach the Earth's surface prior to decay. An approximate zenith angular dependence of cosmic-ray muon intensity is proportional to $\cos ^{2} \theta$ when the curvature of the earth can be neglected $\left(\theta<70^{\circ}\right)$. At large zenith angles, low-energy muons decay before reaching the Earth's surface, and high-energy pions decay be-

Copyright (C) The Society of Geomagnetism and Earth, Planetary and Space Sciences (SGEPSS); The Seismological Society of Japan; The Volcanological Society of Japan; The Geodetic Society of Japan; The Japanese Society for Planetary Sciences; TERRAPUB.

doi:10.5047/eps.2009.06.003 fore interaction, thus increasing the average muon energy level. Thus, cosmic-ray muon radiography can be applied to kilometer-sized objects located at elevations vertically above where the detector is placed. Horizontally arriving cosmic-ray muons are the most suitable particles for radiography of a volcano.

Cosmic-ray muons are particles generated in the atmosphere that continuously bombard the Earth's surface from above. They arrive at angles ranging from the vertical to the horizontal (Gaisser and Stanev, 1998), with an integral intensity of $70 \mathrm{~m}^{-2} \mathrm{~s}^{-1} \mathrm{sr}^{-1}$ at sea level; only an extremely small number of neutrino-induced muons are directed upwards. A high-energy muon can plow through many meters of rock before stopping - the higher the energy, the more rock it can penetrate. Conversely, the more rock, the fewer the muons there will be that have enough energy to get through the rock. For example, a team of physicists and technicians, with oversight from Egyptian archeologists, set up a detection apparatus and associated electronic and computer equipment in the chamber beneath Chephren's pyramid to measure the direction from which a muon comes into one of the burial chambers in the body of the pyramid (Alvarez et al., 1973). The integral nearhorizontal $\left(\theta^{*}=85^{\circ}\right)$ intensity of muons is approximately 
Table 1. Muon range $R$ and energy loss parameters calculated for standard rock. Range is given in km-water-equivalent.

\begin{tabular}{rccc}
\hline $\begin{array}{c}E_{\mu} \\
(\mathrm{GeV})\end{array}$ & $\begin{array}{c}R \\
(\mathrm{~km} . \mathrm{w} . \mathrm{e})\end{array}$ & $\begin{array}{c}a \\
\left(\mathrm{MeV} \mathrm{g}^{-1} \mathrm{~cm}^{2}\right)\end{array}$ & $\begin{array}{c}b \\
\left(\mathrm{MeV} \mathrm{g}^{-1} \mathrm{~cm}^{2}\right)\end{array}$ \\
\hline 10 & 0.05 & 2.15 & 1.91 \\
100 & 0.41 & 2.40 & 3.12 \\
1000 & 2.42 & 2.58 & 4.01 \\
10000 & 6.30 & 2.76 & 4.40 \\
\hline
\end{tabular}

$10^{3}$ and $10^{2} \mathrm{~m}^{-2} \mathrm{sr}^{-1}$ day $^{-1}$ after passing through rock with a thickness of 2000 and 4000 mwe, respectively. It is therefore possible to capture time-sequential images of an active volcano using near horizontal cosmic-ray muons. The volcanic eruption process may result in magma intrusion through a low-density porous conduit and hence be detected via changes in cosmic-ray muon transmission intensity.

Cosmic-ray muon radiography is similar to $\mathrm{X}$-ray radiography, with the exception that penetrating muons serve in place of X-rays. In cosmic-ray muon radiography, a detector with a large surface area $\left(\gtrsim \mathrm{m}^{2}\right)$ is placed at a shallow depth underground with the aim of imaging a volume that elevated with respect to the detector. The intensity of an image pixel in the detector is determined by the attenuation of incident muons caused by absorption in the Earth's crust. The technique is utterly independent on the geophysical model and directly measures the density length $($ density $\times$ path length $)$. By determining the path lengths from topographic information, the measurement provides the average density $\langle\rho\rangle$ along the path lines of cosmic-ray muons through the Earth. This means less rock for muons to penetrate, and more counts from the direction of a lowdensity region. The energy loss of muons through matter can be calculated by exploiting relations in the Standard Model of muon-initiated interactions, which is sufficiently well known for the purpose of radiography (Andreev et al., 1987; Ambrosio et al., 1995; Banerjee et al., 2003). The uncertainty due to theory in these calculations is small. By measuring the muon absorption rate, it is possible to detect a small change in $\langle\rho\rangle$ due to the existence of either less dense or more dense areas inside the volcano. Cosmic-ray muon radiography can be remotely operated. This is a distinct advantage over standard geophysical methods, which must be operated on site, thereby exposing researchers to sometimes difficult or even dangerous (due to volcanic activity) field conditions. The required observation time of the system is inversely proportional to the area of the detector used. For example, the time required for resolving a $3 \%$ change in $\langle\rho\rangle$ in $1 \mathrm{~km}$ of rock is 2 months with a $1,000-\mathrm{cm}^{2}$ detector at solid angle intervals of $0.01 \mathrm{sr}$. Several groups have tried to use this method to see inside a pyramid (Alvarez et al., 1970) and beneath volcanoes (Tanaka et al., 2003, 2005, 2007a, b, c, 2008; Tanaka and Yokoyama, 2008).

The internal structure of the Earth's crust is commonly studied using geophysical observations, such as seismological, electromagnetic, or geodetic measurements. However, these measurements are rather indirect and have substantial intrinsic uncertainties and, therefore, novel and independent tools for assessing subsurface structure are necessary

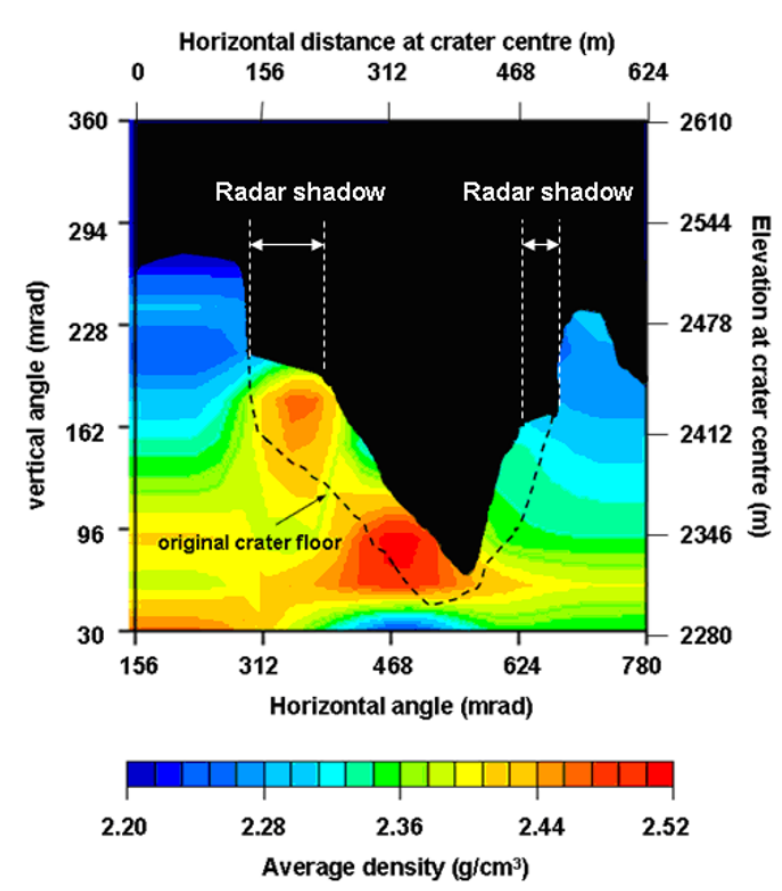

Fig. 1. Reconstructed average density distribution for each muon path line in Mt. Asama. The average density distribution is plotted as a projection on the cross-sectional plane. The distribution of the average density was reconstructed by applying the range-energy relationship for cosmic-ray muons through rock to the path length $(L)$ calculated from the surface shape of the mountain for each arriving angle. The high attenuation region (yellow to red) coincides with the position and shape of the lava mound emplaced in the 2003 crater floor during the 2004 eruption (between white and black dashed lines) with dense lava. A low-density region below (blue) is interpreted as the fall-back of lava in the conduit. The fall-back is consistent with the intermittent collapses of the crater floor that occurred between 22 October 2004 and 11 January 2006. The thickness of the lava mound has been reconstructed from Airborne SAR scanning of the crater floor surface (performed on 11 January 2006).

to reduce these ambiguities. Among the measured properties of the Earth's interior, density plays a special role because it is most readily interpreted in terms of composition and state. These could represent local-scale geological processes, such as the movements of magma in the conduit of a volcano. Thus, independent measurements of density would be of considerable value.

The use of emulsion films (Tanaka et al., 2007a, b, c, 2008; Tanaka and Yokoyama, 2008) has recently made it possible to image the internal structure of a volcano. An emulsion film is a completely power-free particle tracking device that is light enough to be carried up a mountain. Recent technological developments in the particle detector using an emulsion cloud chamber (ECC) (Tanaka et al., 2001) have motivated such radiographic studies of a volcano using a position-sensitive detector designed to detect the particle tracks of cosmic-ray muons with an angular error of a few mrad in the emulsion layers. Using emulsion chambers, Tanaka et al. (2007a, b, 2008) obtained a radiographic image below the crater floor of Mt. Asama with an image resolution significantly higher than would be possible with conventional geophysical measurements. In this study, they found a relatively weak muon-absorption region, indicating 


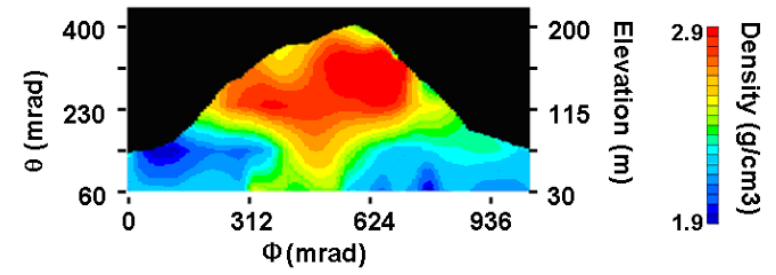

Fig. 2. The average density distribution is plotted as a projection on the cross-sectional plane that is parallel to the detector plane and which includes the 1944 Usu lava dome peak. The distribution of the average density was reconstructed by applying the flux-depth relationship for cosmic-ray muons through rock (Fig. 1) to the path length $(L)$ calculated from the surface shape of the mountain for each horizontal and vertical arriving angle. The right axis is in units of meters corresponding to the elevation of the dome peak.

lower density below the pre-2004 eruption original crater (Fig. 1). One possible physical process that would create such a low-density region below the crater floor is fall-back of fragmented materials into the vent and upper conduit. This fall-back mechanism yields results consistent with the intermittent collapses of the crater floor that occurred between 22 October 2004 and 11 January 2006 (Urabe et al., 2006). After the eruption had ended, the crater floor began to collapse, presumably reflecting the fall-back. Our interpretation of the low-density region is that the fall-back process created the porous magma supply path below the crater floor. The $\langle\rho\rangle$ along the muon path through this region is about $6 \%$ lower than that through the surrounding area at the same depth (Fig. 1). Assuming this porous region is localized in the vent area, it is explained by a conduit diameter of $150 \pm 60 \mathrm{~m}$ (where the error corresponds to the horizontal resolution of the image, which is limited to the statistics available here) with a bulk density of $0.8 \pm 0.2 \mathrm{~g} / \mathrm{cm}^{3}$. This value corresponds to a porosity of $25-45 \%$, inferring a very high permeability. These results indicate that the internal structure inside the erupting crater and vent can be imaged so that it can be inferred whether the vent is plugged with lava or drained to the deeper parts.

To determine whether this method would produce contrasting results when applied to other non-Vulcanian volcanoes, Tanaka et al. $(2007 \mathrm{c}, 2008)$ and Tanaka and Yokoyama (2008) performed cosmic-ray muon radiography in the 1944 Usu lava dome that extrudes as a parasitic cone of Usu, Hokkaido, Japan. They reconstructed the subsurface crustal density structure (Fig. 2) by comparing the transmission image with the local topographic structure, using data on the integrated flux of muons at various zenith angles that penetrated through a given thickness. The radiographic image shown in Fig. 2 is essentially a cross section through the dome parallel to the plane of the detector, on which the average density along all the muon paths is projected. The muon radiography revealed that the lava dome is bulbous and that the magma conduit connected to the dome is less than $100 \mathrm{~m}$ in diameter at its uppermost part. This result proves that the model of the lava dome formation presented in 2002 based on an analysis of its deformation data is approximately correct. The image is drawn with a vertical spatial resolution of $\pm 15 \mathrm{~m}$ and a horizontal resolution of $\pm 15 \mathrm{~m}$. A high-density region can be seen beneath the dome. This region may be explained by a conduit diameter of $100 \pm 15 \mathrm{~m}$ at an elevation of $145 \mathrm{mrad}$ and by a conduit diameter of $50 \pm 15 \mathrm{~m}$ at an elevation of $60 \mathrm{mrad}$. The measurement confirmed a bulbous shape measuring approximately $300 \mathrm{~m}$ in diameter and narrowing in the downward vertical direction. The result is consistent with a model proposed by Yokoyama (Yokoyama, 2002, 2004). The density structure stands in contrast to that of Asama. This knowledge of the subsurface structure of the lava domes is indispensable to any discussion of their formation mechanism. Since completion of the 1944 lava dome, geophysical explorations and deformation analyses of the lava dome have been carried out, but without any convincing results.

Cosmic-ray muon radiography using an emulsion chamber can image such volcanoes and potentially provide a tool for the diagnosis of eruption. However, the image can be obtained only after the emulsion is developed-at a later time-somewhat like a photographic film. Also, largearea emulsions are difficult to scan. In order to overcome this problem, we have developed a portable assembly type cosmic-ray muon telescope module for the real-time imaging of magma movements beneath volcanoes. The results from such studies would be very useful for prediction of upcoming eruptions.

\section{Principle}

Radiography based on the propagation of muons utilizes a well-known energy spectrum for muons arriving at different zenith angles, a technically well-understood muon detector, and a specific muon propagation model through matter. If the Earth's structure along the muon path is unknown, the information from counting muon events in the detector at different arriving angles can be used to infer the properties of the matter through which the muons traveled.

Interactions of very high energy cosmic rays with the atmosphere produce a flux of high-energy cosmic-ray muons, the energy spectrum of which is well known. These muons come mostly from the vertical, following a known zenith angular distribution. However, muons arrive in the horizontal direction as well, with a smaller average intensity but a

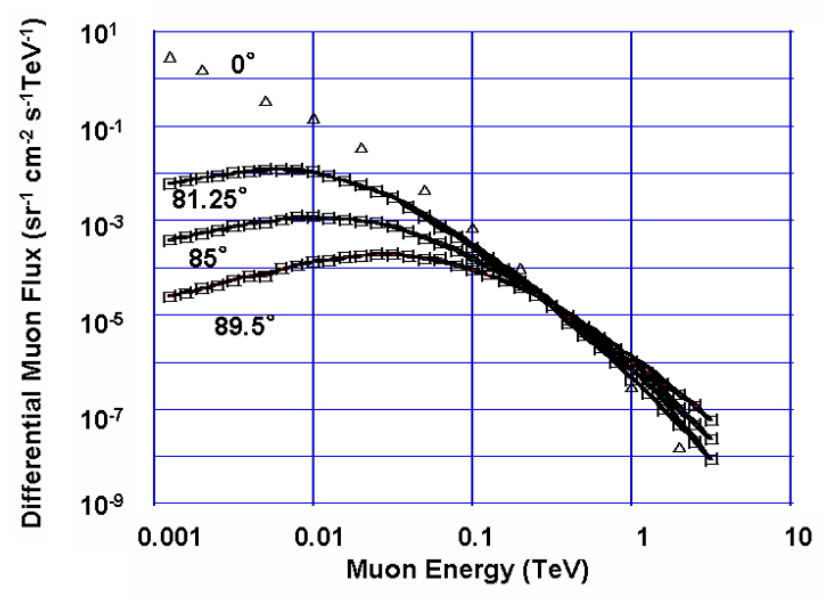

Fig. 3. Energy spectrum of cosmic-ray muons for different zenith angles, where the experimental data are from DEIS (Alkofer et al., 1981), and the curves are the results of the model calculation for large angles. 
higher intensity at energies above a few $100 \mathrm{GeV}$. Because muons become highly penetrative as their energy increases, these horizontal muons can be used for radiographic scanning of the shallow crust. An addition advantage of a muon system is that since the muon $\mathrm{ux}$ is reasonably high, the detector that is required can be kept to a size that is reasonable for geological applications.

First, the energy spectrum of cosmic-ray muons for different zenith angles is obtained from a compilation of experimental data; these data are suf ciently well known for the purpose of radiography. Thompson et al. (1975) assume that the parents of muons are pions and kaons with production spectra of the form $1.8 \times 10^{-6} E^{-2.7}$ (nucleons $\mathrm{cm}^{-2}$ $\left.\mathrm{s}^{-1} \mathrm{Sr}^{-1} \mathrm{TeV}^{-1}\right)$. According to a compilation of the experimental data, the differential muon spectrum $N_{\mu}$ at sea level can be written as follows.

$$
\begin{aligned}
N_{\mu}\left(E_{\mu}, \theta^{*}\right) d E_{\mu}= & 1.8 \times 10^{-6} W_{\mu}\left(E_{\mu}+\Delta E_{\mu}\right)^{-\gamma} \\
& \times\left(\frac{r_{\pi}^{\gamma-1} 90 \mathrm{GeV} \sec \theta^{*}}{E_{\mu}+\Delta E_{\mu}+90 \mathrm{GeV} \sec \theta^{*}}\right. \\
& \left.+0.36 b_{\mathrm{r}} \frac{r_{\mathrm{K}}^{\gamma-1} 442 \mathrm{GeV} \sec \theta^{*}}{E_{\mu}+\Delta E_{\mu}+442 \mathrm{GeV} \sec \theta^{*}}\right) \\
& \left(\mathrm{cm}^{-2} \mathrm{~s}^{-1} \mathrm{sr}^{-1} \mathrm{TeV}^{-1}\right),
\end{aligned}
$$

where $E_{\mu}$ is the muon energy, $\theta^{*}$ is the zenith angle, and $W_{\mu}$ is the survival probability. The ratio of the muon momentum to the momentum of the parent pion $r_{\pi}$ is 0.78 , and the parent kaon $r_{\mathrm{K}}$ is $0.52 . b_{\mathrm{r}}=0.635$ is the branching ratio of the $\mathrm{K}_{\mu 2}$ decay mode. In this equation, the density length of air is $L_{0}=1.013 \mathrm{~kg} / \mathrm{cm}^{2}$, with corresponding muon energy loss $\Delta E_{\mu}=2.6 \mathrm{GeV}$. The survival probability is related to the average decay length $L_{\text {decay }}$ decay of the muon, which is given by:

$$
\begin{aligned}
L_{\text {decay }} & =c \beta \gamma \tau=3 \cdot 10^{8}(\mathrm{~m} / \mathrm{s}) \cdot E(\mathrm{GeV}) / m_{\mu} \cdot 2.2 \cdot 10^{-6}(\mathrm{~s}) \\
& =6.2 \cdot E(\mathrm{GeV}) \mathrm{km},
\end{aligned}
$$

where $\gamma=E_{\mu} / m_{\mu}$ is the Lorentz gamma factor, $c \tau$ (= $660 \mathrm{~m}$ ) is the muon decay length, and $\beta=v / c$. Equation (1) shows the behavior of the muon ux as a function of zenith angle and energy. Figure 3 shows the muon energy spectrum for different zenith angles. At large angles, low-energy muons decay before they interact, thus the average muon energy increases. Solid lines show the theoretical curves for large arriving angles.

The manner in which high-energy muons lose their energy when traveling through matter has been studied well and summarized in various articles (e.g. Adair and Kasha, 1976; Groom, 2001). The energy loss of a charged particle of energy $E$ in tera electron volts (TeV) through matter with a density length of $X$ hectograms per $\mathrm{cm}^{2}\left(\mathrm{hg} / \mathrm{cm}^{2}\right)^{1}$ can be written as:

$$
\mathrm{d} E / \mathrm{d} X=a+b E
$$

where the rst term represents the ionization loss, and the second term represents various stochastic processes $(b=$

\footnotetext{
$\overline{{ }^{1} \mathrm{~A} \text { density length of } 1 \mathrm{hg} / \mathrm{cm}^{2}}$ is equivalent to $1 \mathrm{~m}$ of water (m.w.e).
}

$\left.b_{\mathrm{b}}+b_{\mathrm{p}}+b_{\mathrm{n}}\right)$. At low energies, the energy loss by the ionization process is dominant for all charged particles. However, at suf ciently high energies, radiative processes become more important than ionization. The radiative processes are characterized by the stochastic process, which consists of Bremsstrahlung $\left(b_{\mathrm{b}}\right)$, direct pair production $\left(b_{\mathrm{p}}\right)$, and photonuclear interaction $\left(b_{\mathrm{n}}\right)$. All of these interactions are mediated by the electromagnetic eld of the muon. Cross sections for processes that take place through the weak interaction of the muon with matter are very small and can be neglected. For muons in materials, this critical energy is at an order of several hundred GeV. Table 1 shows $a$ and $b$ values for standard rock (mass number $=22$, proton number $=11$, density $=2.65 \mathrm{~g} / \mathrm{cm}^{3}$ ) as a function of muon energy. The value $a$ has been numerically evaluated for large values of $E$ in standard rock (Adair and Kasha, 1976) as:

$$
a=1.888+0.0768 G \mathrm{MeV} \mathrm{g}^{-1} \mathrm{~cm}^{2},
$$

where $G=\ln \left(E / M c^{2}\right)$. The ionization loss rate per $\mathrm{g} / \mathrm{cm}^{2}$ depends little on the type of the medium. The average energy loss by bremmstrahlung is

$$
\begin{aligned}
& b_{\mathrm{b}}=1.96[G-0.257] \times 10^{-7} \mathrm{~g}^{-1} \mathrm{~cm}^{2},(E<2.5 \mathrm{TeV}) \\
& b_{\mathrm{b}}=1.92 \times 10^{-6} \mathrm{~g}^{-1} \mathrm{~cm}^{2} \text {. } \\
& (E>2.5 \mathrm{TeV})
\end{aligned}
$$

When the energy of the muon is considerably high, a complete screening process predominates. The mean energy loss of a muon to the direct pair production of electronpositron pairs upon passing through $1 \mathrm{~g}$ of material is equal to:

$$
b_{\mathrm{p}}=2.40\left[1-\exp \left(-G^{2} / 40\right)\right] \times 10^{-6} \mathrm{~g}^{-1} \mathrm{~cm}^{2} .
$$

However, direct electromagnetic interactions are not the only causes of energy loss. The nuclear interactions of the muon through the virtual-photon cloud surrounding the muon may also contribute to energy loss. For muons with energy reaching up to dozens of $\mathrm{GeV}$, the nuclear interactions should be accounted for. The cross section for energy-independent photonuclear interactions is approximately given by:

$$
b_{\mathrm{n}}=0.45 \times 10^{-6} \mathrm{~g}^{-1} \mathrm{~cm}^{2} .(E>2.5 \mathrm{TeV})
$$

Once the density length $(X)$ along the path is known, the minimum energy $\left(E_{\mathrm{c}}\right)$ of the cosmic-ray muons which can penetrate through this distance is determined using Eq. (3). By integrating from $E_{\mathrm{c}}$ to in nity, we obtain the integrated muon events $N_{\mu}\left(E_{\mathrm{c}}, \theta^{*}\right)$, which represents the number of muons that have enough energy to escape from the area of interest. Inversely, for a substance with unknown $X$, a measurement of the muon ux $N_{\mu}\left(E_{\mathrm{c}}, \theta^{*}\right)$ uniquely determines its density length, $X$. The angular distribution of the muons in the atmosphere and Digital Terrain Map (DTM) are used to construct the average density along the muon path. The precision of the DTM is usually less than $10 \mathrm{~m}$. Figure 4(a) shows the vertical muon intensity versus depth in units of kilometers of water equivalent (km.w.e). Figure 4(b) shows the integrated ux of near-horizontal cosmic-ray muons, $N_{\mu}\left(E_{\mathrm{c}}, \theta^{*}\right)$, at various zenith angles penetrating through a given thickness of rock, $X$, in units of kilometers of water equivalent. 
a

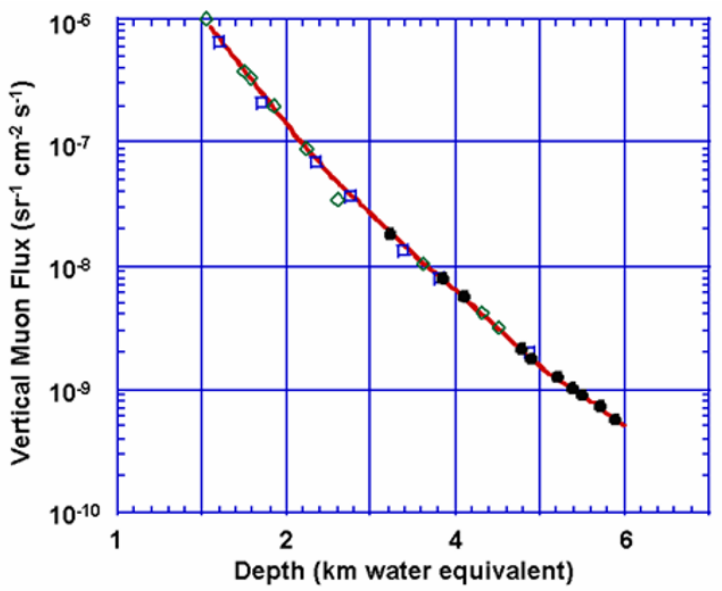

b

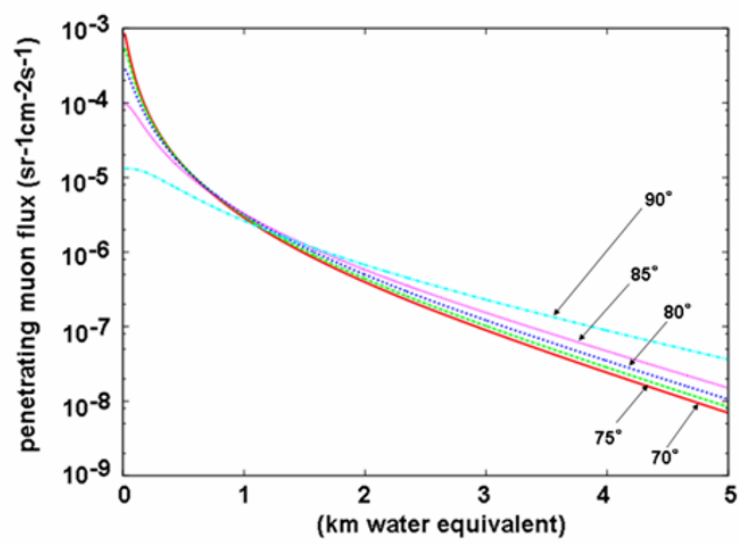

Fig. 4. (a) Vertical muon intensity versus depth $(1 \mathrm{~km}$.w.e. of standard rock). The experimental data are from: $\diamond:$ the compilation of Crouch (Crouch, 1987), $\square$ : Baksan (Andreev, 1987), •: MACRO (Ambrosio et al., 1995). (b) Integrated flux of near-horizontal cosmic-ray muons at various zenith angles penetrating through a given thickness of rock.

\section{Apparatus}

A muon detector requires: (1) the capability to track the arriving angles of cosmic-ray muons; (2) a segmented system comprising assembly-type modules, which enables any problem in the system to be identified by monitoring each module, so that a stable long time operation is possible; (3) the capability to eliminate soft component background (Tanaka et al., 2001).

One possibility is a combination of photomultiplier tubes (PMTs) and plastic scintillators. These devices can be operated under low- and high-temperature conditions. The PMT output consists of a series of electric pulses, with each pulse representing the passage of a muon through the scintillator. Such pulses can be converted to logic pulses and then interpreted as trajectories of muons. The experimental arrangement for a real-time volcano monitoring system requires that: (1) the particle tracking device must be power effective and light enough to be carried up a mountain and (2) the system can be remotely operated. Standard geophysical methods must be operated on site, which can be difficult due to field conditions, or even dangerous due to volcanic activities.

In order to satisfy these two requirements, we have developed a portable assembly-type cosmic-ray muon telescope module. The module consists of a plastic scintillator, an

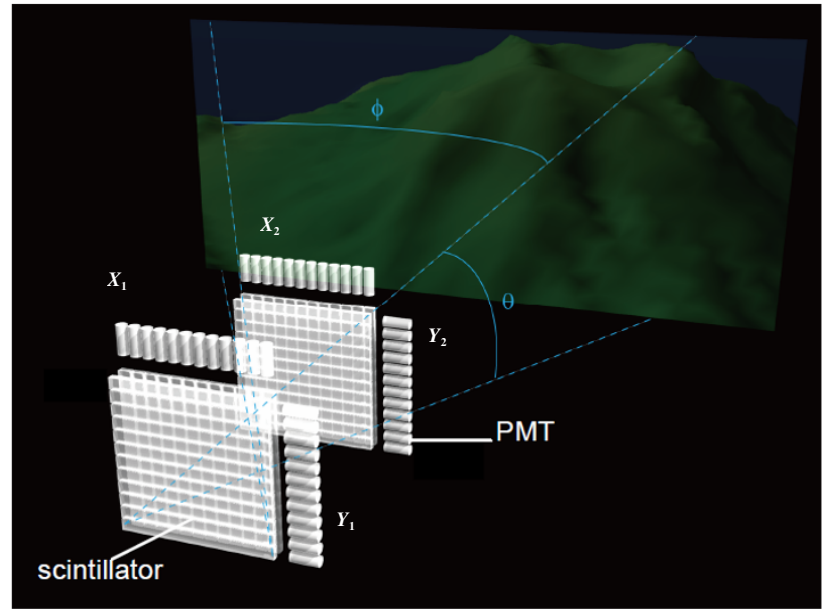

Fig. 5. Portable assembly-type cosmic-ray muon telescope system. The detector matrix counts $12 \times 12$ square pixels of $8 \mathrm{~cm}$.

acryl light guide, and a power-effective PMT (Hamamatsu $\mathrm{H}$ 7724) in a polycarbonate container. Muons are detected by a brief flash of light as they pass through the plastic scintillator. A PMT which provides high photon sensitivity and fast response is widely used as a photosensitive device. Each scintillator is polished and then wrapped in a layer of reflective Mylar and, after being coupled with a PMT, the whole assembly is covered with a light-shielding sheet. This sheet makes the scintillator opaque to external light, internally reflective, and ready for muon detection. Each single module is $150 \times 8 \times 8 \mathrm{~cm}^{3}$ in dimensions and weighs $3.4 \mathrm{~kg} ; 48$ such modules are used to assemble the whole telescope. At the observation site, these 48 modules are arranged so as to set up two segmented scintillation detector planes to track muon trails (a portable assembly-type cosmic-ray muon telescope system). The telescope system consists of crossed segmented scintillator strips (width: $8 \mathrm{~cm}$ ) pointing in the direction of the volcano and allows for the tracking of muons after as pass through the mountain. A straight line connecting the intersecting points of muons at two detector planes (a combination of $X_{1}, Y_{1}$, $X_{2}$, and $Y_{2}$ ) determines the muon trail (Fig. 5). "Forwarddirected" muon trails can be distinguished from "backwarddirected" ones by choosing positive or negative angles, respectively, because muons arriving from below are negligible relative to those arriving from above (i.e., the former are about $10^{-6}$ of the flux of the latter). The intensity of nearhorizontal cosmic-ray muons can be determined from the backward-directed data. Showers and multi-muon events are rejected by discarding events when more than one signal from the same layer (detector plane) coincides in a time gate of $100 \mathrm{~ns}$ (multiplicity cut) (Tanaka et al., 2001).

The muon data are compiled and analyzed by a networkbased FPGA (field programmable gate array) muon readout system. This read-out system comprises powereffective comparators, an FPGA chip, a network chip, and a switching regulator. The total weight of the muon read-out system is $420 \mathrm{~g}$. The direct output from the photomultiplier tube is directed by a $5-\mathrm{m}$ cable to the muon read-out system to convert the PMT voltage spikes into logic pulses deter- 
mined by the transition from a logic-0 to a logic-1 level, which is readable by the FPGA. The FPGA chip computes a muon track by identifying two simultaneous (in $200 \mathrm{~ns}$ ) signals from two different detector planes of the telescope. If the PMT pulses from two detector planes occur within $200 \mathrm{~ns}$, they are deemed to result from a muon passing completely through two detector planes. The gate width of $200 \mathrm{~ns}$ is wide enough for a typical muon-traverse time of $6 \mathrm{~ns}$ between two detectors but narrow enough for a cosmicray muon event rate of $\sim 100 \mathrm{~Hz}$. It is very unlikely that two muon events will occur in the same 200 -ns window. The velocity of light in the plastic scintillator, which has an index of re ection of approximately 1.5 , is $v=c / 1.5$. In addition, the average path that light traverses through the scintillator can be up to $50 \%$ longer than the straight path, due to re ection off the sides of the scintillator with re ective Mylar. This means that for a 1-m scintillator, it takes about $7.5 \mathrm{~ns}$ to travel from one end to the other. As it takes approximately $2 \mathrm{~ns}$ to convert light into an electric current in a PMT, the total delay for the event will be approximately $9.5 \mathrm{~ns}$. This delay is acceptable for the requirements of time resolution.

The PMT signals analyzed in the FPGA chip are recorded in a number of bins representing the horizontal and vertical arriving angles of cosmic-ray muons (Uchida et al., 2010). The histogram is generated on an internal memory of the FPGA as an HTML (HyperText Markup Language)

le. The data according to the address are counted up when an event is detected. These data are read by the network processor when a remote $\mathrm{PC}$ accesses the board. The total power (including a power loss of AC- to DC-conversion) required for the muon read-out system is $5 \mathrm{~W}$, thereby satisfying Conditions 1 and 2.

Because the size of the detector is negligible relative to the length scales of a volcano, we use a coordinate system in which each point on a plane is determined by an angle and a distance (the distance between the detector and the object: $R$ ). The muon transmission image (muon radiograph) is therefore mapped in the angular coordinate. The angular coordinate (also known as the zenith angle or the azimuth angle and denoted by $\theta$ or $\phi$ ) denotes the positive or anticlockwise angle required to reach the point from the $0^{\circ}$ ray. The minimum resolvable distance (spatial resolution: $\Delta X, \Delta Y)$ at an object is de ned by the minimum resolvable angle of the detector. (angular resolution: $\Delta \theta, \Delta \phi$ ) and the distance between the object and the detector $(R)$; $(\Delta X, \Delta Y)=R \times(\Delta \theta, \Delta \phi)$. The angular interval $\left(\theta_{\text {int }}\right)$ and the root mean square angular resolution $\left(\theta_{\text {res }}\right)$ have the following relationship $\left(\theta_{\text {int }}-\theta_{\text {res }}\right)^{2} / \theta_{\text {int }}^{2}=0.32$. Using the data as a function of vertical $(\theta)$ and horizontal $(\phi)$ angles with an angular resolution of $\Delta \theta$ and $\Delta \phi$, we can obtain the value of the density length along the muon path as a function of $\theta$ or $\phi$ with steps of $\Delta \theta$ and $\Delta \phi$. The radiograph is effectively a cross section through the volcano parallel to the plane of the detector on which the average density along all the muon paths is projected.

\section{Results and Discussion}

A preliminary test measurement with the portable assembly type cosmic-ray muon telescope system was performed on campus without a target object, and the results con rmed the integral muon intensity and its zenith-angular dependence (Fig. 6(a)). The whole area of the apparatus is sensitive to muons that come from the near-horizontal plane, but the apparatus does not catch all the muons that enter at large angles to the horizontal. As shown in Fig. 6(a), the telescope has a geometrical ef ciency, which the horizontal distribution of muons can be used to correct. We estimated the systematic error of the detection ef ciency for each arriving angle from the isotropic horizontal distribution of events (Fig. 6(b)). The horizontal distribution at a certain elevation $\left(\theta_{1}\right)$ was normalized to the different horizontal distributions at a different elevations $\left(\theta_{2}\right)$ (normalized events). The experimental error bars were derived by tting a linear function to the backward events and reading deviations of the data from the tting function (Fig. 6(c)). The value was about $6 \%$. A root mean square angular resolution of the system was $\pm 16 \mathrm{mrad}$ at an interval of $40 \mathrm{mrad}$ for a distance between two detector planes of $2 \mathrm{~m}$. The difference between the angular resolution and the interval comes from an ineffective region $( \pm 5 \mathrm{~mm})$ produced between stacked modules. The effective vertical angle is $-320<\theta<640 \mathrm{mrad}$, and the effective azimuth angle is $-480<\phi<480 \mathrm{mrad}$.

Mt. Iwodake, the rhyolitic lava dome on SatsumaIwojima Island (Fig. 7(a)), is located near the northwestern rim of the Kikai caldera (diameter: $\sim 18 \mathrm{~km}$ ), which was formed 6300 years ago. Many fumaroles exist in and around the summit. The chemical composition of volcanic gases from the summit crater of Iwodake has been relatively constant since the 1950s (Kamada, 1964). Isotopic analyses of the composition of the summit volcanic gases indicate that the gases have a magmatic origin (Hedenquist et al., 1994). The degassing of magma requires oversaturation of volatiles in a melt; consequently, degassing occurs only under relatively low-pressure conditions. The highest fumarolic temperature of $900^{\circ} \mathrm{C}$ also supports the idea that volcanic gases were released from the magma at shallow depths. Based on an analogy with the volatile-poor composition of the magma measured in the Showa-Iwojima volcano, it has been estimated that magma degassing occurs in the upper part of Mt. Iwadake (Kazahaya et al., 2002). Thus, it was expected that degassing of a column of magma would be directly observed.

The muon telescope system with a surface area of $1 \mathrm{~m}^{2}$ was installed at an observation point $1.2 \mathrm{~km}$ from the summit crater of Satsuma-Iojima volcano (Fig. 7(b)). The distance between the telescope and the mountain surface was $\sim 500 \mathrm{~m}$, which is much shorter than the muon decay length $(6000 \mathrm{~m})$ for typical muon energy $(1 \mathrm{GeV})$ after passing through the mountain. We therefore did not use iron plates, which is usually necessary for ltering muoninitiated soft-component backgrounds (Tanaka et al., 2001, $2003,2005)$. The total weight of an iron plate with a thickness of 6 radiation lengths is about $1120 \mathrm{~kg}$. It is important to omit this component when making a portable assemblytype muon telescope. The observation altitude was $95 \mathrm{~m}$ a.s.l. It is thought that a magma head resides above the sea level (Kazahaya et al., 2002; Shinohara et al., 2002). Figure 8 shows the counts of muons obtained in a 1-month run with the portable assembly-type cosmic-ray muon tele- 

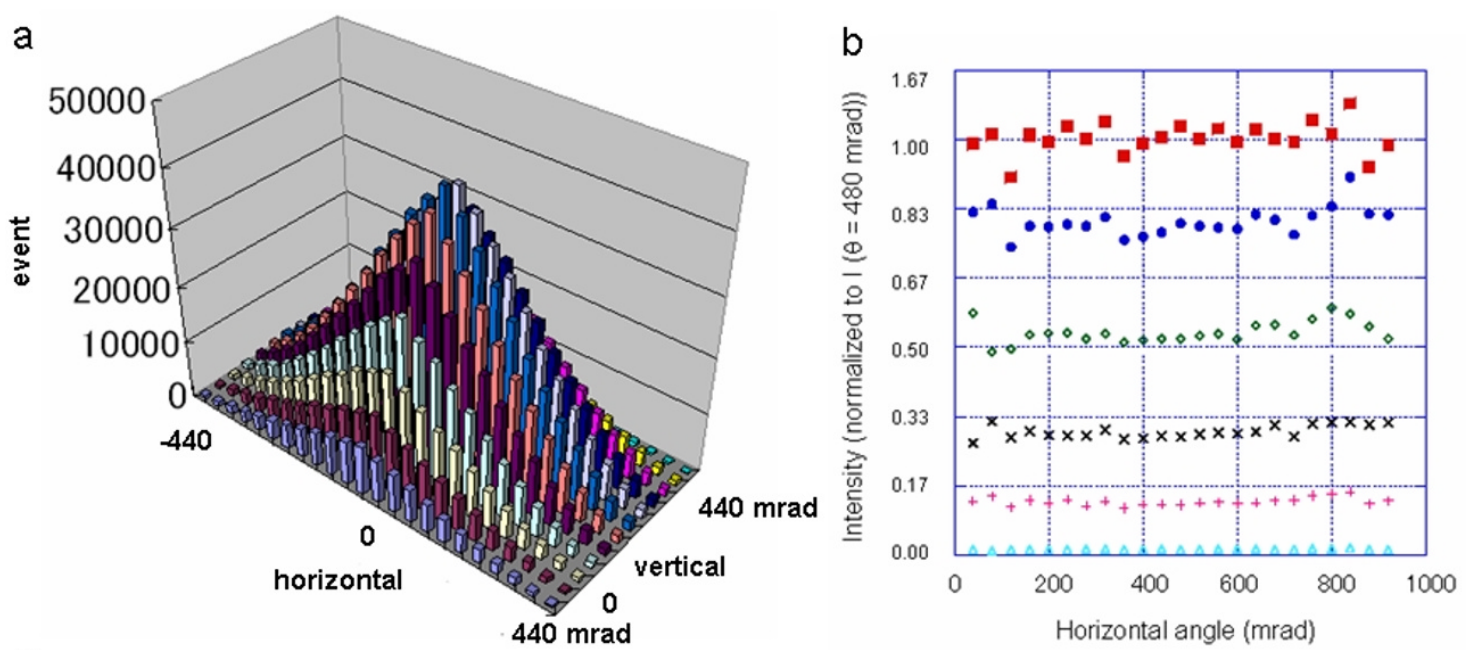

C

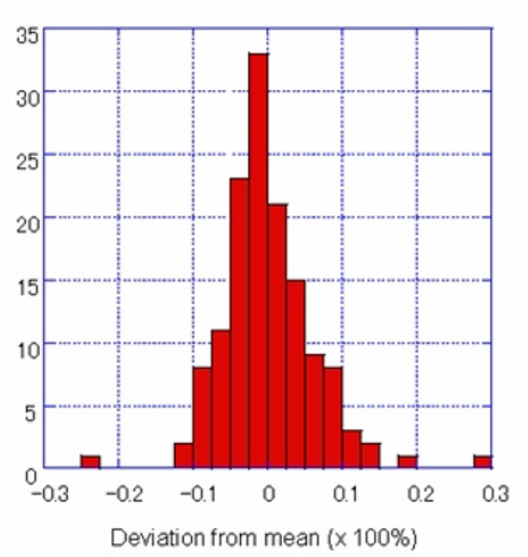

Fig. 6. (a) Typical angular-dependent muon events collected by the muon telescope. (b) Horizontal angle distribution of the normalized events. (c) Distribution of the deviations of the horizontal muon distribution from the fitting function. Statistic errors can be neglected and the deviations are mainly from other sources.

a

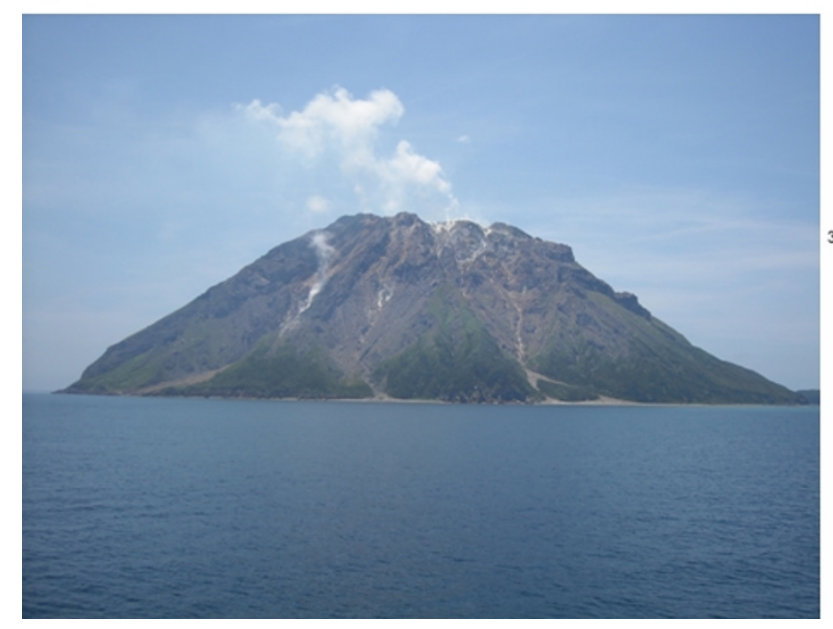

b

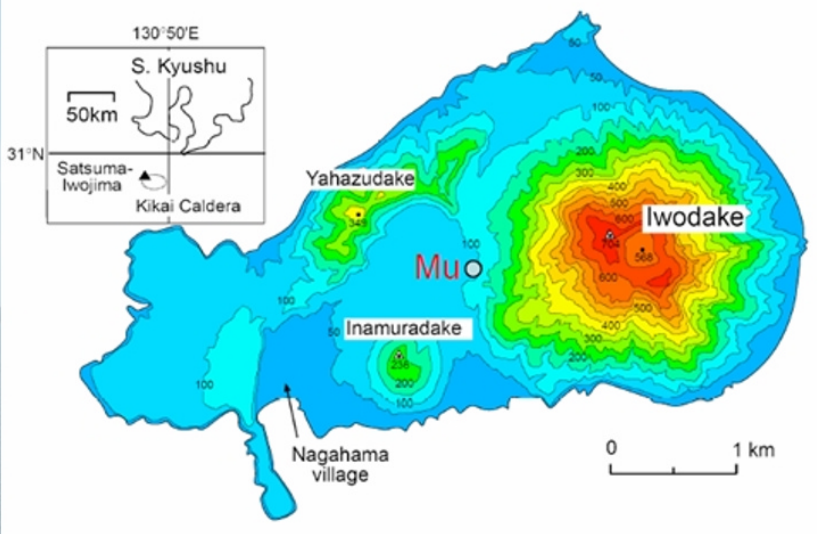

Fig. 7. (a) Photograph of Satsuma-Iojima. (b) Map of Satsuma-Iojima volcano showing the location of the cosmic-ray muon detector (Mu).

scope. The data were packed into the horizontal and vertical bins, which were \pm 56 and \pm 28 mrad, respectively, by taking a moving average at an angular interval of $40 \mathrm{mrad}$. The loss of efficiency at large angles was corrected for. This angular resolution corresponds to the spatial resolution of $\pm 67 \mathrm{mrad}$ in the horizontal direction and $\pm 33 \mathrm{mrad}$ in the vertical direction, with a spatial interval of $48 \mathrm{~m}$ at the crater floor.

During the observation period of the study reported here, $\sim 1.3 \times 10^{6}$ muons sr ${ }^{-1}$ and $\sim 2.0 \times 10^{4}$ muons sr ${ }^{-1}$ were collected for $\theta=400 \mathrm{mrad}$ and $100 \mathrm{mrad}$, respectively. The minimum ionization level for the accumulated pulses 

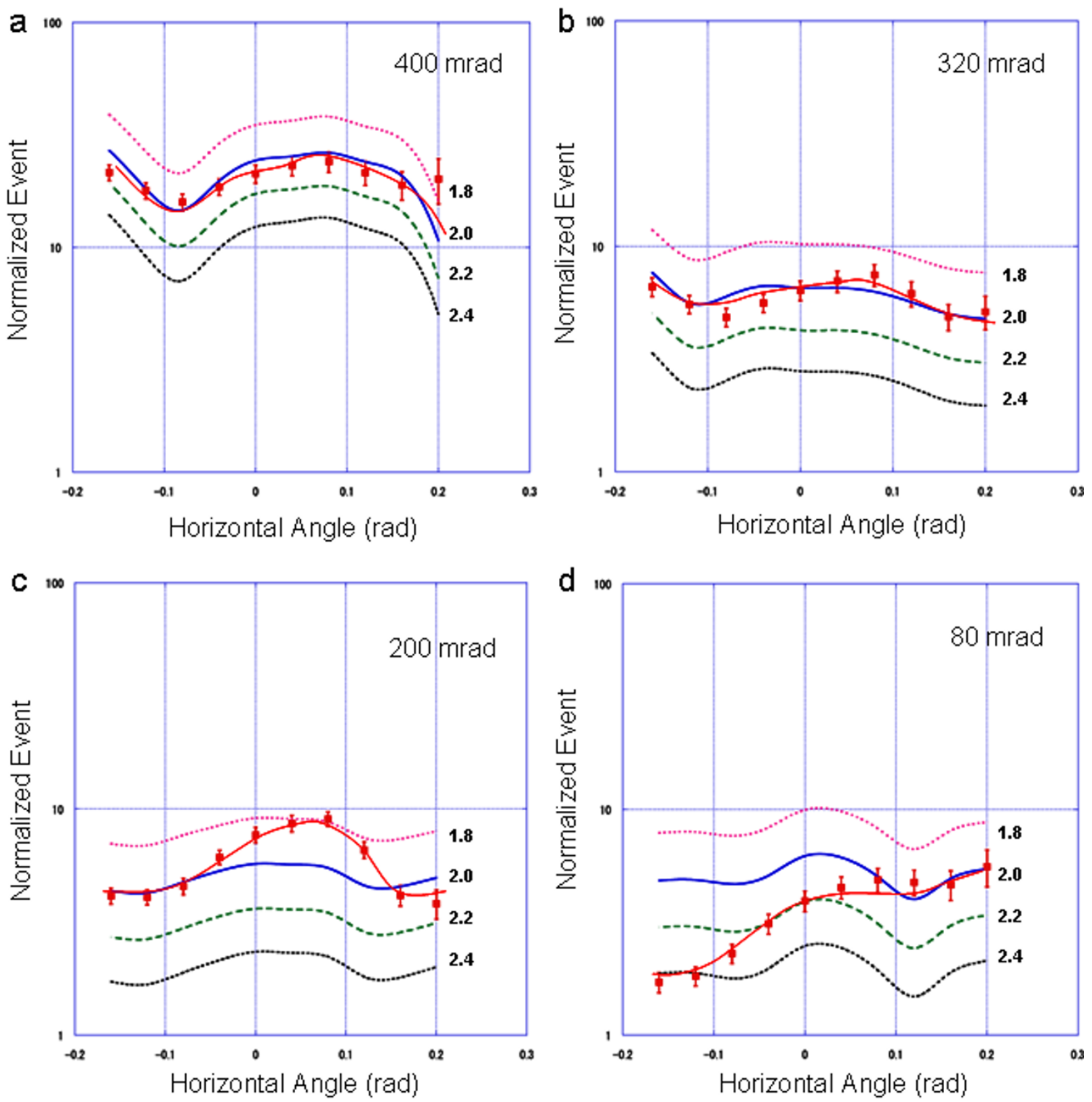

Fig. 8. Counts of muons obtained in a run of 1 month with the portable assembly-type cosmic-ray muon telescope. The number of events is normalized to an observation time of 10 days. The simulation results by referring to the integrated flux of muons at various zenith angles penetrating through a given thickness are also given for the uniform average density value for $\rho=1.8,2.0,2.2$, and $2.4 \mathrm{~g} \mathrm{~cm}^{-3}$.

from each counter was measured by a storagescope. As the value was a few hundred millivolts, the threshold level for each counter was set to be $60 \mathrm{mrad}$, which is sufficiently lower than the minimum ionization level. The single counting rate from each counter was around 10 per second. Those events are mostly coming from the vertical muons that hit the counter with a cross sectional size of $2 \times 100 \mathrm{~cm}^{2}$. We quantify the stronger transmission region by comparing the data integrated over all the observation dates (30 days) with the results of the GEANT4 Monte-Carlo simulations for different uniform average densities. The lines in Fig. 8 show the expected numbers of counts in the same bins, calculated from the geometry of the Mt. Iwodake, the several different densities of the rock, the position of the apparatus, the flux of muons from the sky as a function of angle from the horizontal, and the efficiency of the apparatus as a function of the angle. This calculation assumes there are no density anomalies in the mountain. The experimental error is incorporated into the statistical uncertainty on $N$ counts as a square root of $N$, the standard deviation. The position and the size of the crater in Figs. 8(a-c) are consistent with the GEANT4 Monte-Carlo simulations (solid lines in Fig. 8) (Agostinelli et al., 2003), which give the integrated flux of muons at various zenith angles penetrating through a given density length of rock by referring to the local topographic structure (Tanaka et al., 2007a, b, c).

The stronger muon transmissions come from a longer path length or a higher average density along the path. For $\theta=400 \mathrm{mrad}$, significant increases in events can be seen between $\phi=-0.1$ and $0.2 \mathrm{rad}$ (Fig. 8(a)), primarily due to 


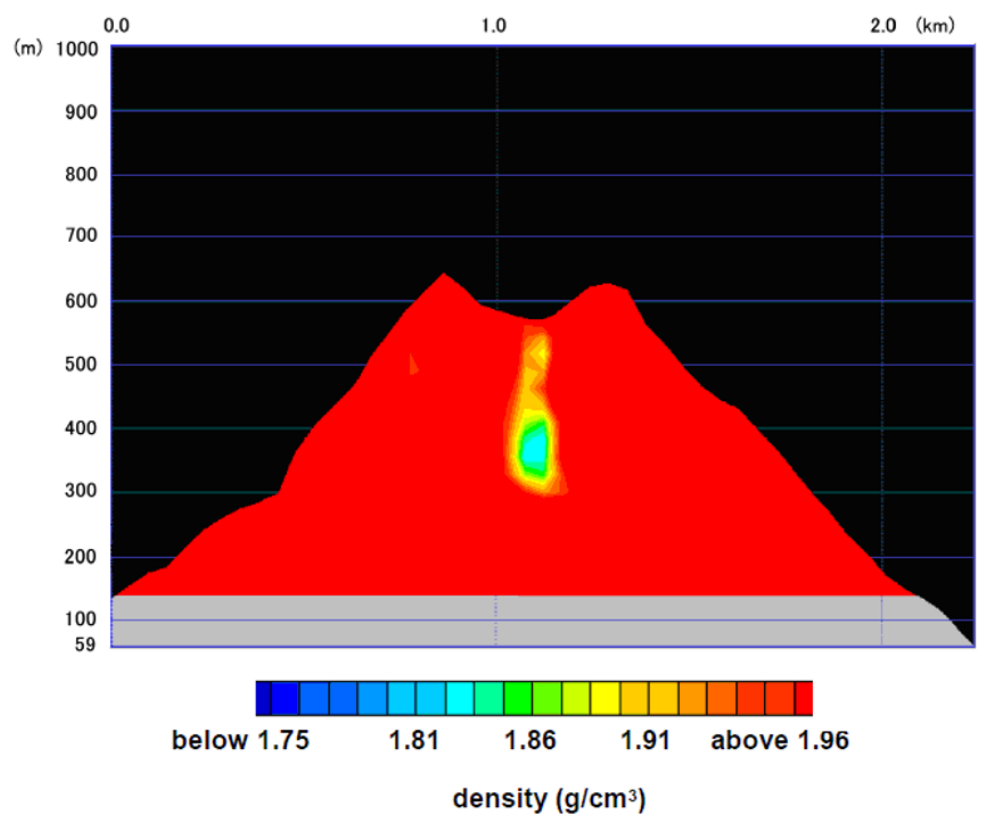

Fig. 9. The average density distribution as a projection on the cross-sectional plane that is parallel to the detector plane and which includes the crater floor of Mt. Iwodake. In order to incorporate the errors in density determination into the image, the density anomalies only below $1.96 \mathrm{~g} \mathrm{~cm}^{-3}$ are mapped.

decreases in the average density along the muon paths in the absence of rock at the top of the mountain (crater). The experimental data are in agreement with the simulation results for $\rho=2.0 \mathrm{~g} \mathrm{~cm}^{-3}$. In the region right below the crater floor $(\theta=320 \mathrm{mrad})$ (Fig. 8(b)), we find a small transmission excess from the simulation line for $\rho=2.0 \mathrm{~g} \mathrm{~cm}^{-3}$ at $\phi \sim 0.08 \mathrm{rad}$. From the comparison with the simulation results, the average density in this region can be derived to $1.92 \mathrm{~g} \mathrm{~cm}^{-3}$. The width of this low-density region is estimated to be $30-50 \mathrm{~m}$. Setting the thickness of the rock at $1000 \mathrm{~m}$ and the average density at $2.0 \mathrm{~g} \mathrm{~cm}^{-3}$, and assuming that this region is localized to the vent area, the local density of this region will become $\rho \sim 0.4 \mathrm{~g} \mathrm{~cm}^{-3}$. In the deeper region $(\theta=200 \mathrm{mrad})$ (Fig. 8(c)) that corresponds to a depth of $200 \mathrm{~m}$ below the crater floor, the size of the excess in the muon transmission intensity from the simulation line for $\rho=2.0 \mathrm{~g} \mathrm{~cm}^{-3}$ becomes larger in the region between $\phi=0$ and $0.1 \mathrm{rad}$. Based on this comparison with the simulation results, the average density in this region is derived to be $1.82 \mathrm{~g} \mathrm{~cm}^{-3}$. The width of this low-density region is estimated as $\sim 200 \mathrm{~m}$. Setting the thickness of the rock at $1550 \mathrm{~m}$ and the average density at $2.0 \mathrm{~g} \mathrm{~cm}^{-3}$ and assuming that this density anomaly is localized to the vent area, we obtain a local density of this region of $\rho \sim 0.9 \mathrm{~g} \mathrm{~cm}^{-3}$. However, in the region located further deeper in the conduit $(\theta=80 \mathrm{mrad})$ (Fig. 8(d)), which corresponds to a depth of $280 \mathrm{~m}$ below the crater floor, the amount of muon transmission decreases. Since muon radiography only resolves the average density distribution along the muon paths, we must assume a more localized structure along the muon path. On the other hand, it is reasonable to assume that the observed density variations are localized in the crater area because volcanoes are axi-symmetric. We there assumed that the density deviation in Fig. 8(c) is localized in the crater area and that the density deviation in Fig. $8(\mathrm{~d})$ is from the heterogeneity of the body of Iwodake.

Figure 9 shows the average density distribution projected on the cross-sectional plane that is parallel to the detector plane and which includes the crater floor. The value of the experimental error $(2.5 \%$ in determining the density length) was evaluated from the systematic error of the detection efficiency for each arriving angle. The error derived by estimating the path length using the topographic map $(1 / 25000)$ may in some cases be as high as $30 \mathrm{~m}$ compared to the actual $1500-\mathrm{m}$ path length, which is a $2.0 \%$ error. Therefore, the total density length relative error becomes $3.2 \%$. Figure 8 shows two low-density regions below the crater floor: (a) a region right below the crater floor and (b) a larger region below Region (a). Region (a) may be interpreted as the low-density fragmented materials in the upper conduit. Below Region (b) is a higher density region that we interpret to be the top of a magma column (Region (c)). The density of this region is close to the surrounding density $\left(2.1-2.2 \mathrm{~g} \mathrm{~cm}^{-3}\right)$, indicating non-degassed magma with bubbles.

We can now construct a picture of the amount of muon transmission through Mt. Iwodake in order to interpret our data and arrive at an estimate for the degassing activity in the conduit. Our picture of the various depths is as follows.

(1) The region between 0 and $100 \mathrm{~m}$ below the crater floor: this region may consist of low-density collapsed materials forming a high-temperature fumarolic area at the center of the crater. The pathway seems to have a diameter of $30-50 \mathrm{~m}$. The volcanic gas is emitted through this porous area when a magma conduit extends to a shallow level and is allowed to degas.

(2) The region between 100 and $300 \mathrm{~m}$ below the crater 


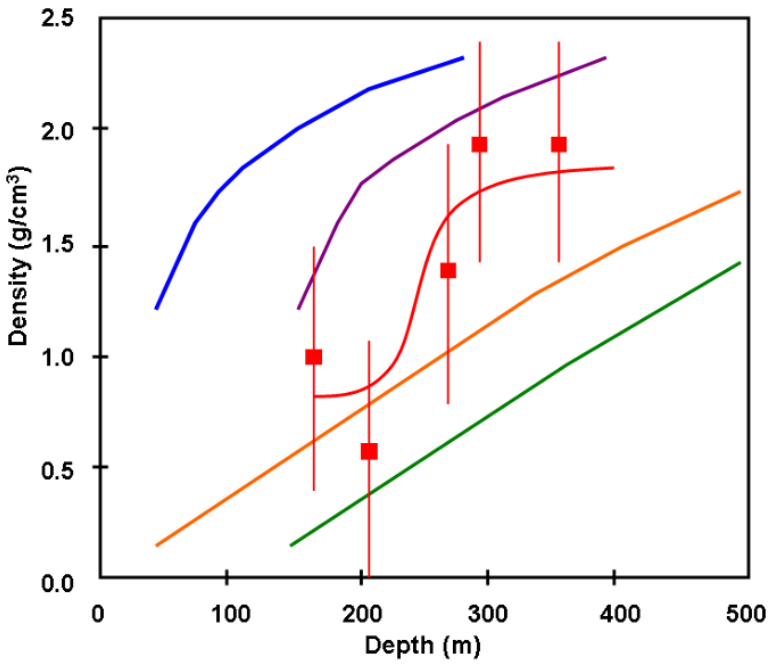

Fig. 10. One-dimensional density modeling in the conduit together with the density distribution as obtained from the muon observation. The red line is drawn through the data points to guide the eye. The orange and blue lines correspond to the densities of ascending and descending magma, respectively, when the magma head is located at a place $280 \mathrm{~m}$ below the crater floor. The green and purple lines correspond to the densities of ascending and descending magma, respectively, when the magma head is located at a place $150 \mathrm{~m}$ below the crater floor.

floor: this region may consist of ascending extremely low density undegassed magma caused by bubble expansion and coalescence in magma. In this region, the uppermost part of the magma degases efficiently, and the degassed magma descends through the region around the low-density magma, as shown in Fig. 2(d). The diameter of this convection region seems to be $\sim 200 \mathrm{~m}$.

(3) The region deeper than $300 \mathrm{~m}$ below the crater floor: this region may consist of ascending undegassed magma column. The depth of the magma head observed $300 \mathrm{~m}$ below the crater floor is consistent with the degassing pressure of magma of $0.5-3.0 \mathrm{MPa}$ in Satsuma-Iojima, as estimated by laboratory and theoretical studies (Kazahaya et al., 2002; Shinohara et al., 2002).

Based on Fig. 9, we can interpret that there are two cylindrical low-density regions: (1) the region between the crater floor and a position $150 \mathrm{~m}$ below the crater floor, and (2) the region between positions at $150 \mathrm{~m}$ and $280 \mathrm{~m}$ below the crater floor. These regions can be explained either as (1) a layer filled with pumice rocks or (2) magma that contains many bubbles. Because Iwojima is not erupting at the present time it is unlikely that the magma head reaches the crater floor. It is therefore assumed that there are two possible locations of the magma head: (1) $150 \mathrm{~m}$ below the crater floor, and (2) $280 \mathrm{~m}$ below the crater floor. The condition of the conduit can be modeled by assuming (1) a degassed magma density of $2.4 \mathrm{~g} \mathrm{~cm}^{-3}$, (2) an initial $\mathrm{H}_{2} \mathrm{O}$ density of $1.5 \mathrm{wt} \%$, (3) a pressure gradient of $25 \mathrm{MPa} \mathrm{km}^{-1}$, (4) a surrounding density of $2.0 \mathrm{~g} \mathrm{~cm}^{-3}$, (5) a conduit diameter of $160 \mathrm{~m}$, (6) magma that holds bubbles when it ascends, and (7) magma descent when the void ratio of magma becomes $50 \%$ at $1 \mathrm{MPa}$. We also consider that the desolution of $\mathrm{H}_{2} \mathrm{O}$ into magma when it descends. One-dimensional density modeling in the conduit is shown in Fig. 10 for the different positions of the magma head in the conduit together with the density distribution as obtained from the muon observation. Since the average density in a conduit depends on the ratio of ascending and descending magma, the density deduced by muon radiography must be in between the ascending and descending curves. The orange and blue lines in Fig. 10 correspond to the densities of ascending and descending magma when the magma head is located at a place $280 \mathrm{~m}$ below the crater floor, respectively. Green and purple lines correspond to the densities of ascending and descending magma when the magma head is located at a place $150 \mathrm{~m}$ below the crater floor, respectively. If we assume that the magma head is located at a place $150 \mathrm{~m}$ below the crater floor, the low-density region, as visualized at the center of the image (blue patch in Fig. 9), can be explained by magma with many bubbles.

\section{Conclusion}

The data presented here provide clear evidence that we have imaged the degassing of a column of magma in the volcanic conduit of Satsuma Iojima volcano within 1 week. This technique only resolves the average density distribution along individual muon paths. Therefore, at the end of the day, the user must always make assumptions or interpretations about the more localized structure along the muon paths, or he/she must use more than one detector to resolve the three-dimensional density structure. This is why volcanoes make good study targets: they are axi-symmetric, and it is reasonable to assume that the observed density variations are localized in the vent or crater area. The method is limited to depths above sea level and strongly depends on the nature of the local topography (the detector must be placed at a location, in the direction of a topographically prominent feature of interest, and there will only be results for the volume above the detector). However, the measurement yields information on anomalies in the density distribution, such as from high- or low-density magma.

The measurements provide the perfect data for studying the local density structure in the Earth's crust at sites which cannot be well resolved because of their strong structural heterogeneity and potential difficulty to be accessed, and which therefore cannot have their structure determined by conventional electromagnetic or seismic techniques. The duration of monitoring in this study was limited to 1 month in order to confirm if the method was applicable for nearfuture time-dependent measurement with a time interval of about 1 month.

Acknowledgments. Special funding arrangements by S. Okubo, K. Nakatsuka, and associated researchers of the ERI, JSPS (Japanese Society of Promotion of Science) and JST (Japan Science and Technology Agency) are acknowledged. M. Takeo and T. Koyaguchi of ERI, K. Nagamine of UCR, I. Yokoyama, M.J.A. H. Imabeppu, Y. Yamashina, and S. Steigerwald are also acknowledged for their valuable suggestions.

\section{References}

Adair, R. K. and H. Kasha, Muon Physics, edited by V. W. Hughes and C. S. Wu, vol. 1, p. 323, Academic Press, 1976. 
Agostinelli, S., J. Allison, K. Amako, J. Apostolakis, H. Araujo, P. Arce, M. Asai, D. Axen, C. O. Allkofer et al., Muon spectra from DEIS up to 7 TeV, Proc. 17th Cosmic Ray Conf. (Paris), 10, 321, 1981.

Agostinelli, S. et al., Geant4: A simulation tool kit, Nucl. Inst. Meth. A, 506, 250-303, 2003.

Allkofer, C. O. et al., Muon spectra from DEIS up to $7 \mathrm{TeV}$, Proc. 17th Cosmic Ray Conf. (Paris), 10, 321, 1981.

Alvarez, L. W. et al., Search for hidden chambers in the pyramids, Science, 167, 832-839, 1970.

Ambrosio, M. et al. (MACRO collaboration), Vertical muon intensity measured with MACRO at the Gran Sasso laboratory, Phys. Rev., D52, 3793-3802, 1995.

Andreev, Yu. M., V. I. Gurentzov, and I. M. Kogai, in Proc. 20th Int. Cosmic Ray Conf. (Moscow), 6, 200, 1987.

Banerjee, S., G. Barrand, F. Behner, L. Bellagamba, J. Boudreau, L. Broglia, A. Brunengo, H. Burkhardt, S. Chauvie, J. Chuma, R. Chytracek, G. Cooperman et al., Geant4: A simulation tool kit, Nucl. Inst. Methods. A, 506, 250-303, 2003.

Crouch, M., in Proc. 20th Int. Cosmic Ray Conf. (Moscow), 6, 165, 1987.

Gaisser, T. K. and T. Stanev, Cosmic rays, review of particle physics, Eur. Phys. J. C, 3, 132-137, 1998.

Groom, D. E., Muon stopping power and range tables $10 \mathrm{MeV}-100 \mathrm{TeV}$, Atomic Data and Nuclear Data Tables, 78, 183-356, 2001.

Hedenquist, J. W., M. Aoki, and H. Shinohara, Flux of volatiles and oreforming metals from the magmatic-hydrothermal system of Satsuma Iwojima volcano, Geology, 22, 585-588, 1994.

Kamada, M., Volcano and geothermy of Iwo-jima, Kagoshima prefecture, Jinetsu, 3, 1-23, 1964 (in Japanese).

Kazahaya, K., H. Shinohara, and G. Saito, Degassing process of SatsumaIwojima volcano, Japan: Supply of volatile components from a deep magma chamber, Earth Planets Space, 54, 327-335, 2002.

Shinohara, H., K. Kazahaya, G. Saito, N. Matsushima, and Y. Kawanabe, Degassing activity from Iwodake rhyolitic cone, Satsuma-Iwojima volcano, Japan: Formation of a new degassing vent, 1990-1999, Earth Planets Space, 54, 175-185, 2002.

Tanaka, H. K. M. and I. Yokoyama, Muon radiography and deformation analysis of the lava dome formed by the 1944 eruption of Usu, Hokkaido-Contact between high-energy physics and volcano physics-, Proc. Jpn. Acad., Ser B, 84, 107-116, 2008.

Tanaka, H., K. Nagamine, N. Kawamura, S. N. Nakamura, K. Ishida, and K. Shimomura, Development of the cosmic-ray muon detection system for probing internal-structure of a volcano, Hyperfine Interactions, 138, 521-526, 2001.
Tanaka, H., K. Nagamine, N. Kawamura, S. N. Nakamura, K. Ishida, and K. Shimomura, Development of a two-fold segmented detection system for near horizontally cosmic-ray muons to probe the internal structure of a volcano, Nucl. Instr. Methods. A, 507, 657-669, 2003.

Tanaka, H. K. M., K. Nagamine, S. N. Nakamura, and K. Ishida, Radiographic measurement of the internal structure of Mt. West Iwate with near-horizontal cosmic-ray muons and future developments, $\mathrm{Nucl}$. Instr. Methods. A, 555, 164-172, 2005.

Tanaka, H. K. M., T. Nakano, S. Takahashi, J. Yoshida, and K. Niwa, Development of an emulsion imaging system for cosmic-ray muon radiography to explore the internal structure of a volcano, Mt. Asama, Nuclear Instr. Methods Phys. Res. A, 575, 489-497, 2007a.

Tanaka, H. K. M., T. Nakano, S. Takahashi, J. Yoshida, M. Takeo, J. Oikawa, T. Ohminato, Y. Aoki, E. Koyama, H. Tsuji, and K. Niwa, High resolution imaging in the inhomogeneous crust with cosmic-ray muon radiography: The density structure below the volcanic crater oor of Mt. Asama, Japan, Earth Planet. Sci. Lett., 263, 104-113, 2007 b.

Tanaka, H. K. M., T. Nakano, S. Takahashi, J. Yoshida, H. Ohshima, T. Maekawa, H. Watanabe, and K. Niwa, Imaging the conduit size of the dome with cosmic-ray muons: The structure beneath Showa-Shinzan Lava Dome, Japan, Geophys. Res. Lett., 34, L22311, 2007c.

Tanaka, H. K. M., T. Nakano, S. Takahashi, J. Yoshida, M. Takeo, J. Oikawa, T. Ohminato, Y. Aoki, E. Koyama, H. Tsuji, H. Ohshima, T. Maekawa, H. Watanabe, and K. Niwa, Radiographic imaging below a volcanic crater oor with cosmic-ray muons, Am. J. Sci., 308, 843-850, 2008.

Thompson, M. G. et al., The production spectra of the parents of vertical cosmic ray muons, J. Phys. G: Nucl. Phys., 1, L48-L50, 1975.

Uchida, T., H. K. M. Tanaka, and M. Tanaka, Development of a muon radiographic imaging electronic board system for a stable solar power operation, Earth Planets Space, 62, this issue, 167-172, 2010.

Urabe, B., N. Watanabe, and M. Murakami, Topographic change of the summit crater of Asama volcano during the 2004 eruption derived from Airborne Synthetic Aperture Radar (SAR) measurements, Bull. Geogr. Surv. Inst., 53, 1-6, 2006.

Yokoyama, I., Growth mechanism of the 1944 lava dome of Usu volcano in Hokkaido, Japan, Proc. Jpn. Acad. 78, Ser. B, 6-11, 2002.

Yokoyama, I., Formation processes of the 1909 Tarumai and the 1944 Usu lava domes in Hokkaido, Japan, Ann. Geophys., 47, 1811-1825, 2004.

H. K. M. Tanaka (e-mail: ht@riken.jp), T. Uchida, M. Tanaka, H. Shinohara, and H. Taira 